\title{
Using k-Nearest Neighbor Algorithm to Identify Mechanical Vibrational Modes of a Cantilever with Spectrally Multiplexed Fiber Bragg Gratings
}

\author{
Senta L. Jantzen ${ }^{1, *}$, Jiarui Yu ${ }^{2}$, Peter G. R. Smith ${ }^{1}$ and Christopher Holmes ${ }^{1}$ \\ 1 Optoelectronics Research Centre, University of Southampton, SO17 1BJ, UK \\ 2 Information and Electronic Engineering College of Zhejiang University, Zhejiang, China \\ *Corresponding author: S.Jantzen@soton.ac.uk
}

\begin{abstract}
We demonstrated for the first time the identification of mechanical modes of a cantilever with attached fiber Bragg gratings using k-Nearest Neighbor, a machine learning algorithm. We analyzed the frequency range of $40-300 \mathrm{~Hz}$ and an acceleration of $1.1 \pm 0.1 \mathrm{~g}$. (C) 2020 The Author(s)
\end{abstract}

\section{Introduction}

Machine learning algorithms learn from experience and make decisions without being specifically programmed. In engineering applications, they are often used for condition monitoring to predict failure of mechanical systems. This reduces unexpected downtime of equipment and lowers risk of potential structural damage [1], [2]. In condition monitoring, operational changes are typically identified using a threshold value [3], here we use a k-Nearest Neighbor (kNN) approach and fiber Bragg gratings (FBGs).

FBGs are ideal for structural health monitoring, as they can be implemented as quasi-distributed sensors. The shift in peak wavelength is typically used for sensing. Here, we explore small changes in the spectral bandwidth and shape of the grating reflections and apply machine learning to extract information about the mechanical modes, which can be used for failure prediction.

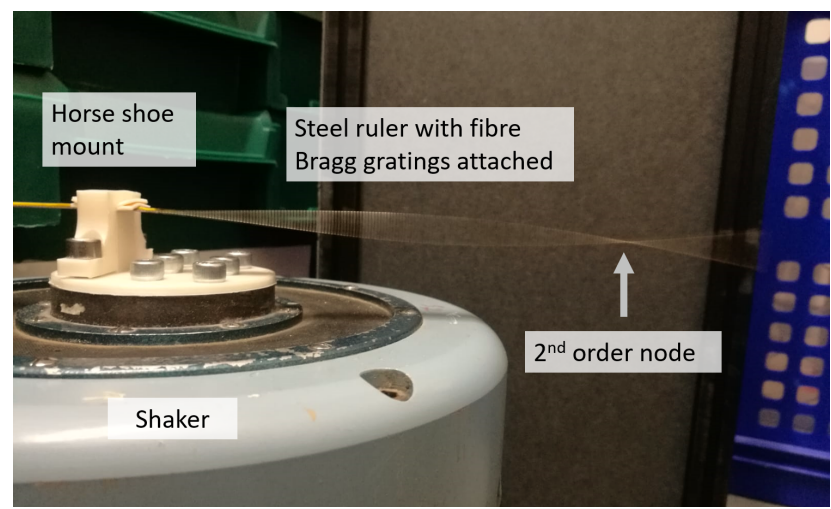

Fig. 1. Experimental set up: Steel ruler as cantilever with fiber Bragg gratings attached is mounted on a shaker. The cantilever is driven in the second order mode with a higher acceleration amplitude for demonstration.

\section{Experimental Procedure and Results}

This work demonstrates the identification of mechanical vibration modes of a $300 \mathrm{~mm}$ long cantilever with the kNN algorithm. Fig. 1 shows the set up with FBGs taped on a ruler, mounted on one side and placed on a shaker (Derritron VP4). A training data set, which forms data clusters for certain events is required to set up a kNN algorithm. In this case, the four events are 'no vibration', $2^{\text {nd }}, 3^{\text {rd }}$ and $4^{\text {th }}$ order mode, which were calculated to occur at $0 \mathrm{~Hz}, 52 \mathrm{~Hz}, 146 \mathrm{~Hz}$ and $287 \mathrm{~Hz}$. The shaker was driven at an acceleration amplitude of $1.1 \pm 0.1 \mathrm{~g}$. The $\mathrm{kNN}$ algorithm used for this work calculates the euclidean distance between a test point and the $k$ nearest points of the previously recorded training data set. The most commonly occurring neighbors then determine classification. 
A total of 28 Bragg gratings, each $5 \mathrm{~mm}$ in length, were inscribed using small spot direct UV writing [4]. The training data set consists of 50 optical spectra each for every mechanical mode. The gratings respond differently to vibration, depending on their physical location on the ruler and the location of the mechanical nodes. As a measure of the spectral bandwidth, the spectral spread was calculated for each grating bin:

$$
\text { Spread }=\frac{\int R d \lambda}{\max |R|}
$$

with reflected power $\mathrm{R}$ in $\mathrm{mW}$ and the central Bragg wavelength $\lambda$ in $\mathrm{nm}$.

A testing data set was recorded by sweeping the drive frequency of the shaker and capturing an optical spectrum for each frequency from $40-300 \mathrm{~Hz}$ with a step size of $1 \mathrm{~Hz}$. The clustering of the training data set as well as the data points from the sweep are shown in Fig. 2 a). Each of the four events form a distinct cluster in the 3-dimensional environment, which indicates the possibility to classify using $\mathrm{kNN}$.

a)

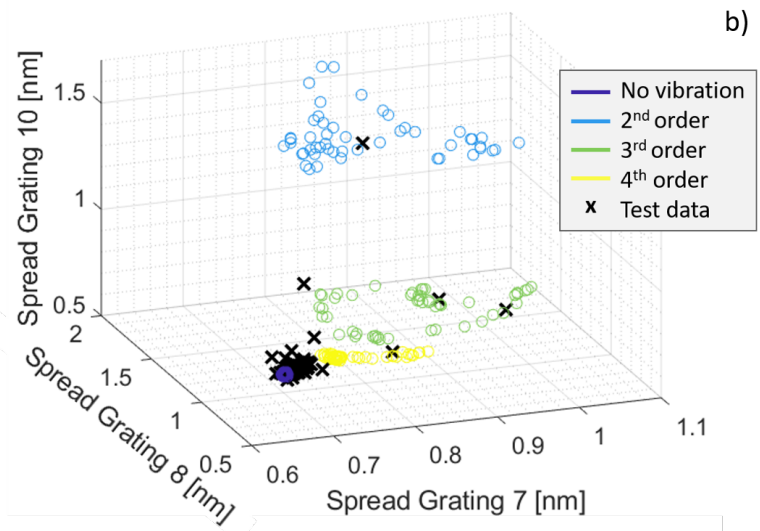

b)

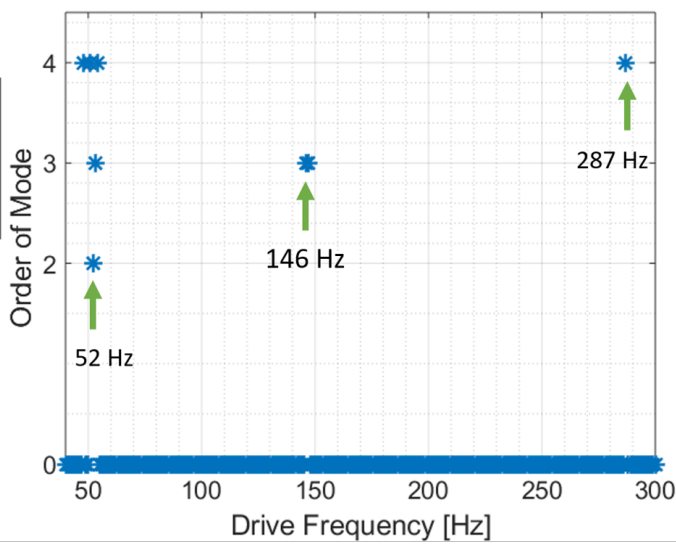

Fig. 2. a) Training data forms clusters for the four cases: no vibration, $2^{\text {nd }}, 3^{\text {rd }}, 4^{\text {th }}$ order mode. b) The output of the $\mathrm{kNN}$ algorithm with $\mathrm{k}=5$. The green arrows show the correct classification for the different modes.

The result of the testing data for $\mathrm{k}=5$ nearest neighbors is shown in Fig. 2 b). As indicated by the green arrows, the mechanical modes are correctly classified, however the algorithm incorrectly classified frequencies slightly above and below the $2^{\text {nd }}$ order mode to $3^{\text {rd }}$ and $4^{\text {th }}$ order mode. The spectral response of the second order mode is so large compared to the other modes, that on approach to the $2^{\text {nd }}$ order mode cluster the spectral features appear similar to the $3^{\text {rd }}$ and $4^{\text {th }}$ order mode, which leads to a misclassification.

We will investigate a variation of different mechanical drive amplitudes to determine the limitations of this approach. We will also explore circular features in Fig. 2 a), which we believe are birefringence artifacts.

\section{Conclusion}

This is the first demonstration of identifying mechanical modes of a cantilever using fiber Bragg gratings and the k-Nearest Neighbor algorithm. We analyzed a drive frequency range between $40-300 \mathrm{~Hz}$ at an acceleration of $1.1 \pm 0.1 \mathrm{~g}$. We demonstrated the classification of the $2^{\text {nd }}, 3^{\text {rd }}$ and $4^{\text {th }}$ order mode and will further investigate the effect of varying acceleration amplitudes and analyze the limitations of this classification method.

\section{References}

1. Stetco, A., Dinmohammadi, F., Zhao, X., Robu, V., Flynn, D., Barnes, M., Nenadic, G., ”Machine learning methods for wind turbine condition monitoring: A review.", Renewable Energy, 133, 2019.

2. Li, S., Sun, L., "Detectability of Bridge-Structural Damage Based on Fiber-Optic Sensing through DeepConvolutional Neural Networks". Journal of Bridge Engineering, 25(4), 2020.

3. Antoniadou, I., Worden, K. "Use of a spatially adaptive thresholding method for the condition monitoring of a wind turbine gearbox", 7th European Workshop on Structural Health Monitoring, 2014.

4. Scholl, S. L., Jantzen, A., Bannerman, R. H. S., Gow, P. C., Smith, D. H., Gates, J. C., Holmes, C., "Thermal approach to classifying sequentially written fiber Bragg gratings. Optics Letters", 44(3), 2019. 